\title{
New Theory of Light and Resolution of the Abraham-Minkowski Controversy
}

\author{
Adnan Salih AL-ITHAWI \\ Department of Physics, Huazhong University of Science and Technology, Wuhan 430074, China
}

('Corresponding author's e-mail: dradnan_salih@yahoo.com)

Received: 25 August 2018, Revised: 30 July 2019, Accepted: 20 August 2019

\begin{abstract}
The Abraham-Minkowski controversy about the momentum of light in media has been debated for over a century and has been informed by many distinguished distributions, both theoretical and experimental. We show that both the Abraham and Minkowski forms of momentum are need to be modified according to the new theory of light. We prove that the total momentum of a photon in matter is the same as compared with that in free space by using the new relations between energy and mass of light with refractive index.
\end{abstract}

Keywords: Momentum, Controversy, Mass, Energy, Photon, Abraham-Minkowski

\section{Introduction}

Since the introduction of the photon hypothesis by Planck in 1900 [1], the relationship between the energy (E) and momentum $(P)$ of a photon propagating in a vacuum has been known to be $(E=P C)$. Momentum is the most important parameter in physics. The conservation law of momentum is universal. According to the special theory of relativity, a photon has momentum, but has no mass, and this is very surprising, for how can something have momentum without mass? One can review the study of the momentum of photons, sometimes called radiation pressure, over the century in the form of a series of connected topics which have converted the experiments and theoretical studies. The number of theoretical papers is as vast as 25 times the number of experimental papers [2]. The momentum of a light wave propagating in a medium has remained the subject of extensive controversy until now [3]. This controversy is known as the Abraham-Minkowski dilemma [4]. The rivaling momenta of light in a medium are $\left(\mathrm{P}_{\mathrm{A}}=\mathrm{E} / \mathrm{nc}\right)$ (Abraham) [5,6] and $\left(\mathrm{P}_{\mathrm{M}}=\mathrm{nE} / \mathrm{c}\right)$ (Minkowski) [7], where (n) is the refractive index. To explain the ambiguities, it has also been suggested that both forms of momenta are correct but simply represent different aspects of photon momentum [8,9]. The Abraham-Minkowski controversy in some other works has been claimed to have been resolved by arguing that the division of the total energymomentum tensor into electromagnetic and material components would be arbitrary [10-12]. Barnett concluded in his resolution that the Abraham version is the kinetic momentum, and the Minkowski version is the canonical momentum; "the kinetic momentum of a body is simply the product of its mass and velocity", while "the canonical momentum of a body is simply Planck's constant divided by its de Broglie wavelength" [13]. Wang concluded that a photon takes Abraham momentum in the Einstein-box thought experiment, while it takes Minkowski momentum in the photon-atom Doppler resonance absorption experiment, with both Abraham and Minkowski momenta being correct photon momenta [14].

In this study, we will try to resolve this controversy by using the principles of a new theory of light $[15,16]$ which suggest that the energy of a photon is changeable in medium but does not stay constant, as in the current theory, and also suggests that a photon has natural mass in a vacuum, not 0 rest mass, again as in the current theory, effectively having mass in medium. 
http://wjst.wu.ac.th

\section{Theoretical analysis}

As we know, the momentum of a photon in vacuum is [7];

$\mathrm{P}=\mathrm{E}_{\mathrm{vc}} / \mathrm{C}$

For Minkowski relationship, the momentum of a photon in mediumis;

$\mathrm{P}=\mathrm{nE}_{\mathrm{med}} / \mathrm{C}$

We think that the difference in the computational value of a photon's momentum in Eqs. (1) and (2) is due to the value of energy of the photon in vacuum and medium. Experimentally, we proved that $[15,16]$;

$\mathrm{E}_{\mathrm{vc}} \neq \mathrm{E}_{\mathrm{med}}$

We found, theoretically, according to the principles of the new theory $[15,16]$, that the value of energies is different by a factor of the refractive index;

$\mathrm{E}_{\mathrm{vc}}=\mathrm{nE}_{\mathrm{med}}$

where $\left(E_{\mathrm{vc}}\right)$ is the energy of a photon in vacuum, and $\left(\mathrm{E}_{\mathrm{med}}\right)$ the energy of a photon in medium.

Experimentally, we proved that the value of kinetic energy of a photon inside the vapor of water decreased by a factor of the refractive index, as in Eq. (4) [17]. We measured the value of the kinetic energy of a photon in water and silica glass in medium, and found that the value decreased by the refractive index also [18]. This means that Eq. (4) is correct. If we use Eq. (4) in Eq. (2), we get;

$\mathrm{P}=\mathrm{E}_{\mathrm{vc}} / \mathrm{C}$

Equation (5) is the same as Eq. (1); this mean that the momentum of a photon is constant in vacuum and medium.

For Abraham relationship, the momentum of photon in medium is;

$\mathrm{P}=\mathrm{E} / \mathrm{nc}$

Abraham supposed the photon was a particle and derived his relation from [5];

$\mathrm{P}=\mathrm{mc}$

$\mathrm{P}=\mathrm{mv}$

Where (m) in Eq. (7) is the mass of a photon in vacuum, and (m) in Eq. (8) is the mass of a photon in medium. Is the value of the mass of a photon the same as in vacuum and medium? According to current theories (particle and wave), a photon is massless in vacuum. We suggested a theoretical form $[15,16]$, where a photon has mass in vacuum $\left(\mathrm{m}_{\text {nat }}\right)$, and effective mass $\left(\mathrm{m}_{\text {eff }}\right)$ in medium; we proved theoretically that value of masses is different by a factor of the refractive index $[15,16]$;

$\mathrm{m}_{\mathrm{eff}}=\mathrm{n} \mathrm{m}_{\text {nat }}$ 
http://wjst.wu.ac.th

Where $\left(\mathrm{m}_{\text {nat }}\right)$ is the mass of a photon in vacuum, while $\left(\mathrm{m}_{\text {eff }}\right)$ is the mass of a photon in medium. As we know the relation between mass and energy of a photon is [19];

$$
\begin{aligned}
& \mathrm{E}=\mathrm{m}_{\text {nat }} \mathrm{c}^{2} \\
& \mathrm{~m}_{\text {nat }}=\mathrm{E} / \mathrm{c}^{2} \\
& \mathrm{~m}_{\text {nat }}=\mathrm{h} / \mathrm{c} \lambda_{\mathrm{o}}
\end{aligned}
$$

Where $\left(E=h v\right.$, and $\left.v=c / \lambda_{\mathrm{o}}\right)$, equation $(12)$ can be rearranged;

$\mathrm{h} / \mathrm{c}=\mathrm{m} \cdot \lambda_{\mathrm{o}}$

It is difficult to measure the mass of a photon, but there is another parameter called the mass length of a photon in vacuum $(\mathrm{Y}),[20]$;

$\mathrm{Y}=\mathrm{m} \cdot \lambda_{\mathrm{o}}$

From Eqs. (1) and (14);

$\mathrm{Y}^{*}=\mathrm{m}_{\mathrm{eff} \cdot \lambda} \lambda$

where $\left(\mathrm{Y}^{*}\right)$ is the mass length of a photon in medium.

According to the new theory, the wavelength of a photon is constant in vacuum and medium $\left(\lambda_{\mathrm{o}}=\right.$ $\lambda)$.

From Eqs. (1), (14), and (15);

$\mathrm{n}=\mathrm{Y}^{*} / \mathrm{Y}$

where (n) is the refractive index.

Let us consider a laser $(532 \mathrm{~nm})$, chopped by $(4 \mathrm{~mm})$. The number of photons $\left(\mathrm{N}_{\mathrm{ph}}\right)$ in this beam is;

$\mathrm{N}_{\mathrm{ph}}=\mathrm{L} / \lambda_{\mathrm{o}}$

$\mathrm{N}_{\mathrm{ph}}=4 * 10-3 / 532 * 10-9=7518$ photons.

For the laser beam $(532 \mathrm{~nm})$, the natural mass from Eq. (10) is;

$\mathrm{m}_{\text {nat }}=4.15^{*} 10^{-33} \mathrm{~kg}$

Theoretically, when this laser beam with length $(4 \mathrm{~mm})$ enters glass with a refractive index $(1.5261)$ [3], from Eq. (1), the effective mass $\left(\mathrm{m}_{\mathrm{eff}}\right)$ is;

$\mathrm{m}_{\mathrm{eff}}=6.3394194 * 10^{-33} \mathrm{~kg}$

From Eqs. (14) and (15);

$\mathrm{Y}=6.62607004 * 10-34 / 3 * 10^{8}=2.208690013 * 10^{-42} \mathrm{~kg} . \mathrm{m}$.

$\mathrm{Y}^{*}=6.3394194 * 10^{-33} * 532 * 10^{-9}$

$\mathrm{Y}^{*}=3.37 * 10^{-42} \mathrm{~kg} . \mathrm{m}$ air is;

Now, if we have a laser beam $(532 \mathrm{~nm})$, and a number of photons $(7518)$, the length of the beam in 
$\left(\mathrm{L}_{\text {air }}=\mathrm{Y} * \mathrm{~N}_{\mathrm{ph}} / \mathrm{m}_{\text {nat }}\right)$ :

$\mathrm{L}_{\text {air }}=2.208690013 * 10^{-42} * 7518 / 4.15^{*} 10^{-33}$

$\mathrm{L}_{\mathrm{air}}=4 \mathrm{~mm}$

When this laser beam with length $(4 \mathrm{~mm})$ enters glass $(\mathrm{n}=1.5261)$, the length of the beam will be;

$\mathrm{L}_{\mathrm{med}}=(\mathrm{Y} * \mathrm{Y}) * \mathrm{~L}_{\mathrm{air}}$

$\mathrm{L}_{\text {med }}=6.1 \mathrm{~mm}$

When the laser enters the surface of the glass at an angle, let the length of the laser beam on the surface be $\left(\mathrm{L}_{\text {air }}=4 \mathrm{~mm}\right)$. According to the current theory, the wavelength of a photon in vacuum $(\lambda \mathrm{o})$ (e.g., $532 \mathrm{~nm})$ when entering a glass medium $(\mathrm{n}=1.5261)$; the wavelength $(\lambda)$;

$\lambda=\lambda \mathrm{o} / \mathrm{n}$

$\lambda=348.6 \mathrm{~nm}$

If we have (7815) wavelength or photon, the length of laser beam $\left(\mathrm{L}_{\text {med }}\right)$ is;

$\mathrm{L}_{\text {med }}=348.4 * 7815=2.7 \mathrm{~mm}$

Now, which is correct: the current theories, or the new theory?

\section{Experimental}

A laser light with wavelength $(532 \mathrm{~nm})$ was used, which broke a glass window with a refractive index of (1.5261) [3]. Part of the laser beam entered the front surface of the glass, and another part entered the glass as in Figure 1.

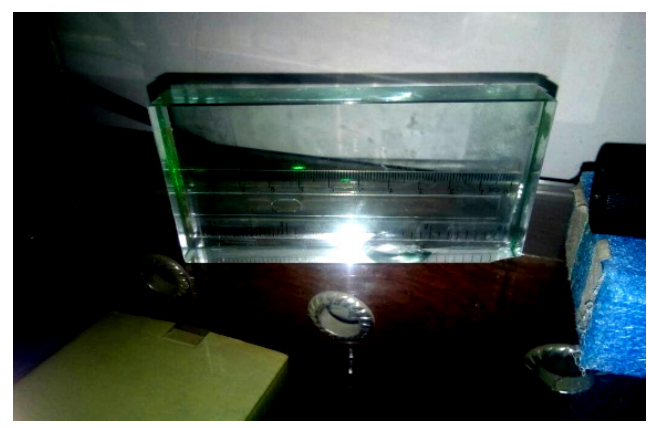

(a) Experimental setup

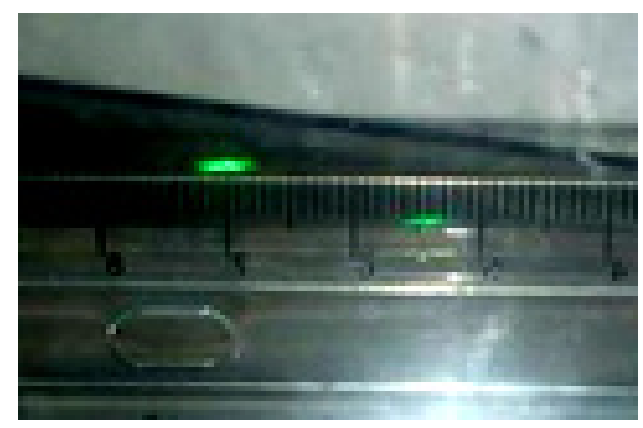

(b) Result of experiment

Figure 1 Part of the laser beam entered the front surface of the glass, and another part entered the glass; (a) Experimental setup, and (b) Result of experiment.

From Figure 1, the laser beam length, experimentally, was $6.1 \mathrm{~mm}$ in medium and $4 \mathrm{~mm}$ in air. The results of the experiment are summarized in Table 1.

Table 1 The measured length and mass length of a laser beam in air and medium. 
http://wjst.wu.ac.th

\begin{tabular}{ccccccc}
\hline $\mathbf{\Lambda 0}$ & $\mathbf{m}_{\text {nat }} \mathbf{~ k g}$ & $\mathbf{L}_{\text {air }}$ & $\mathbf{Y}$ & $\mathbf{n}_{\mathbf{g}}$ & $\mathbf{Y}^{*}$ & $\mathbf{L}_{\text {med }}$ \\
\hline $532 \mathrm{~nm}$ & $4.154 * 10^{-33}$ & $4 \mathrm{~mm}$ & $2.209 * 10^{-42} \mathrm{~kg} \cdot \mathrm{m}$ & 1.5261 & $3.37 * 10^{-42} \mathrm{~kg} \cdot \mathrm{m}$ & $6.1 \mathrm{~mm}$ \\
\hline
\end{tabular}

From Eqs. (16) and (18);

$\mathrm{n}=\mathrm{L}_{\text {med }} / \mathrm{L}_{\text {air }}$

Where (n) is the refractive index of the glass. According to Eq. (20);

$\mathrm{n}=6.1 / 4$

$\mathrm{n}=1.525$

Which is approximately the same as in [3]. This proved that the new theory is correct, as in Eq. (17).

If we use Eq. (17) in Eq. (16), and we derive the momentum as Abraham did, we find that;

$\mathrm{P}=\mathrm{E} / \mathrm{C}$

Equation (10) is the same as Eq. (1).

This means that the momentum of a photon is constant in vacuum and medium.

\section{Conclusions}

In this study, we used the relations of a new theory of light to solve the controversy of AbrahamMinkowski about the momentum of light in medium. Minkowski dealt with the photon as a wave with relation according to the current theory, which suggests that the energy of a photon is constant in vacuum and medium; therefore, he found the momentum increased by a factor of the refractive index in medium, but we proved that the energy of a photon decreased by a factor of the refractive index in medium. We substituted the value of energy in medium $\left(E_{\text {med }}=E_{a v} / n\right)$ in the relation of Minkowski; the result was that the momentum in medium was the same as in vacuum. We did the same with the Abraham relation, which deals with the photon as a particle; we proved also that the momentum of a photon is the same in vacuum and medium.

\section{References}

[1] M Planck. Zur theorie des Gesetzes der Energieverteilung im Normalspectrum. Verh. Dtsch. Phys. Ges. 1900; 2, 237.

[2] C Baxter and R Loudon. Radiation pressure and the photon momentum in dielectrics. J. Mod. Optics 2010; 57, 830-42.

[3] M Partanen, T Hayrynen, J Oksanen and J Tulkki. Photon mass drag and the momentum of light in a medium. Phys. Rev. A 2017; 95, 063850.

[4 ME Crenshaw. Decomposition of the total momentum in a linear dielectric into field and matter components. Ann. Phys. 2013; 338, 97-106.

[5] M Abraham. Zur Elektrodynamik bewegter Körper. Rend. Circ. Matem. Palermo. 1909; 28, 1.

[6] M Abraham. Sull'elettrodinamica di Minkowski. Rend. Circ. Matem. Palermo. 1910; 30, 33.

[7] H Minkowski. Die Grundgleichungen fur die elektromagnetischen Vorgange in bewegten Korpern. Math. Ann. 1910; 68, 472-525.

[8] U Leonhardt. Abraham and Minkowski momenta in the optically induced motion of fluids. Phys. Rev. A 2014; 90, 033801. 
http://wjst.wu.ac.th

[9] PW Milonni and RW Boyd. Momentum of light in a dielectric medium. Adv. Opt. Photon 2010; 2 , 519-53.

[10] RNC Pfeifer, TA Nieminen, NR Heckenberg, and H Rubinsztein-Dunlop. Constraining validity of the Minkowski energy-momentum tensor. Phys. Rev. A 2009; 79, 023813.

[11] PJ Penfield and HA Haus. Electrodynamics of Moving Media. MIT Press, Cambridge, 1967.

[12] SRD Groot and LG Suttorp. Foundations of Electrodynamics. North-Holland, Amsterdam, 1972.

[13] SM Barnett. Resolution of the Abraham-Minkowski Dilemma. Phys. Rev. Lett. 2010; 104, 070401.

[14] C Wang. Self-consistent theory for a plane wave in a moving medium and light-momentum criterion. Can. J. Phys. 2015; 93, 1510-22.

[15] A Salih. Mass, energy, and momentum of photon in medium. Int. J. Phys. Sci. 2013; 18, 1190-2.

[16] AS Al-Ithawi. The photon as a mass particle associated with wave. Int. J. Optic. Appl. 2015; 5, 225.

[17] AS Al-Ithawi and AH Ali. Experimentally proved that, the refractive index effected on energy of photon in medium. Int. J. Optic. Appl. 2015; 5, 151-4.

[18] J Asmaa, K Al-Kinani, IM Kadhim and AS Al-Ithawi. A new technique for measuring the refractive index. Int. J. Nanoelectron. Mater. 2018; 11, 427-34.

[19] A Einstein. E= $\mathrm{Mc}^{2}$. Ideas and Opinion. Dell, New York, 1976, p. 330.

[20] Photon Energy, Mass, Velocity and Wavelength - The Living Universe. http://www.circlon.com/home/7-Photon-Energy.html, accessed May 2018. 